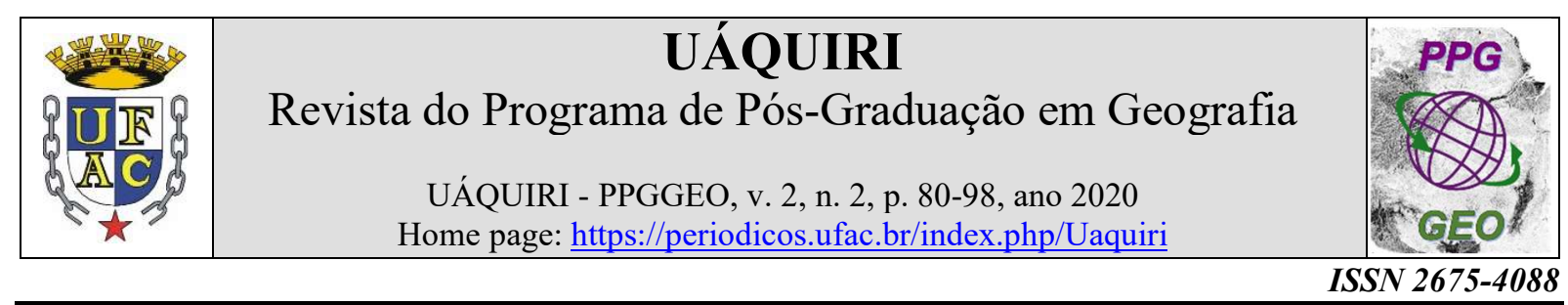

\title{
VARIAÇÃO GRANULOMÉTRICA E MORFOLÓGICA DOS SEDIMENTOS DE FUNDO DO RIO ACRE: CONTRIBUIÇÕES INICIAIS AO ESTUDO HIDROSSEDIMENTOLÓGICO DE CANAL FLUVIAL NO SUDOESTE AMAZÔNICO
}

Fábio da Silva Lucio ${ }^{1 *}$, Gabriel Brandão Xavier ${ }^{1}$, Ms. Anderson Azevedo Mesquita ${ }^{1,2}$, Dr. José Genivaldo do Vale Moreira ${ }^{3,5}$, Dr. Waldemir Lima dos Santos ${ }^{1,4}$,

Dr. Rodrigo Otávio Peréa Serrano ${ }^{1,5}$

ORCID: https://orcid.org/0000-0002-7273-7648; https://orcid.org/0000-0001-8071-1862; https://orcid.org/0000-0002-2994-8482; https://orcid.org/0000-0002-5306-5612; https://orcid.org/0000-0002-5306-5612; https://orcid.org/0000-0002-7786-8305

${ }^{1}$ Universidade Federal do Acre (UFAC), cursos de Geografia Bacharelado, Rio Branco, Acre; ${ }^{2}$ Doutorando do Programa de pós-graduação em geografia da Universidade Federal de Rondônia, Porto Velho, Rondônia; ${ }^{3}$ Professor da UFAC, Campus Floresta, Cruzeiro do Sul, Acre, Brasil; ${ }^{4}$ Professor do programa de pós-graduação em Geografia da UFAC, Rio Branco, Acre. ${ }^{6}$ Professor do programa de pós-graduação em Ciência, Inovação e

Tecnologia para a Amazônia da UFAC, Rio Branco Acre. fabiogeografoac@gmail.com

Recebido em: 02/06/2020 Aceito em: 23/09/2020. Publicado em: 20/12/2020 DOI: https://doi.org/10.47418/uaquiri.vol2.n2.2020.3803

\begin{abstract}
RESUMO
O ciclo hidrosedimentológico é de fundamental importância para a compreensão da dinâmica fluvial e modelagem do ambiente físico. Em relação ao rio Acre, sua dinâmica geomorfológica está ligada ao deslizamento das margens do rio, o que obedece às variações do regime fluvial com as cheias e vazantes. Os estudos morfológicos são um importante indicativo do comportamento hidrodinâmico, bem como, os fatores externos que influenciam: rocha matriz, ambiente deposicional, agentes envolvidos e durabilidade das partículas. O presente trabalho tem por objetivo analisar a variação granulométrica do leito do rio Acre nos municípios de Assis Brasil, Brasiléia e Rio Branco, como também, através da aplicação de técnicas de processamento de imagem associadas ao cálculo e interpretação de índices morfométricos, quantificar a variabilidade dimensional da fração areia presente no leito fluvial do rio Acre, nas amostras da cidade de Rio Branco. Por fim, o trabalho conclui que: i) o tamanho médio dos grãos dos sedimentos de fundo do rio Acre, vão diminuído de diâmetro ao longo do trajeto da cidade de Assis Brasil a Rio Branco; ii) a técnica de morfometria de sedimentos mostrou evidências do processo de desgaste e remobilização das partículas desde a saída da rocha matriz até sua deposição; iii) o uso do software ImageJ se mostrou eficiente na velocidade e precisão para análise e diferenciação morfometrica dos sedimentos arenosos no trecho analisado.
\end{abstract}

Palavras-chave: Dinâmica fluvial. Granulometria de sedimentos. Morfometria. Rio Acre. Sedimentologia. 


\title{
GRANULOMETRIC AND MORPHOLOGICAL VARIATION OF ACRE RIVER BOTTOM SEDIMENTS: INITIAL CONTRIBUTIONS TO THE HYDRO-SEDIMENTOLOGICAL STUDY OF THE RIVER CANAL IN THE SOUTHWESTERN AMAZON
}

\begin{abstract}
The hydrosedimentological cycle is of fundamental importance for the understanding of river dynamics and modelling of the physical environment. In relation to the Acre River, its geomorphologic dynamics is linked to the river banks sliding, which obeys the variations of the fluvial regime with the floods and vazantes. Morphological studies are an important indication of hydrodynamic behavior, as well as the external factors that influence: matrix rock, depositional environment, agents involved and durability of particles. The purpose of this article is to analyze the particle size variation of the acre river bed in the municipalities of Assis Brazil, Brasileia and Rio Branco, as well as, through the application of image processing techniques associated with the calculation and interpretation of morphometric indices, to quantify the dimensional variability of the sand fraction present in the river bed of the Acre River, in the samples of the city of Rio Branco. Finally, the work concludes: i) the average grain size of the bottom sediments of the Acre river is reduced in diameter along the route from the city of Assis Brazil to Rio Branco; ii) the sediment morphometry technique showed evidence of the process of wear and remobilization of the particles from the exit of the matrix rock to their deposition; iii) the use of ImageJ software proved efficient in speed and precision for analysis and morphometric differentiation of the sandy sediments in the analyzed stretch.
\end{abstract}

Keywords: Fluvial dynamics; Sediment granulometry; Morphometry; Acre river; Sedimentology.

\section{VARIACIÓN GRANULOMÉTRICA Y MORFOLÓGICA DE LOS SEDIMENTOS DE FUNDO DO RIO ACRE: CONTRIBUCIONES INICIALES AL ESTUDIO HIDROSEDIMENTOLÓGICO DEL CANAL FLUVIAL EN LA AMAZONAS SUROESTE}

\begin{abstract}
RESUMEN
El ciclo hidrosedimentológico es de fundamental importancia para la comprensión de la dinámica del río y el modelado del entorno físico. En relación con el río Acre, su dinámica geomorfológica está vinculada al deslizamiento de tierra del río, que obedece a las variaciones del régimen fluvial con las inundaciones y los reflujos. Los estudios morfológicos son un indicador importante del comportamiento hidrodinámico, así como, de los factores externos que influyen: roca matriz, ambiente de depósito, agentes involucrados y durabilidad de las partículas. El presente trabajo tiene como objetivo analizar la variación granulométrica del lecho del río Acre en las ciudades de Assis Brasil, Brasiléia y Rio Branco, así como, mediante la aplicación de técnicas de procesamiento de imágenes asociadas con el cálculo e interpretación de índices morfométricos, para cuantificar la variabilidad. dimensión de la fracción de arena presente en el cauce del río Acre, en las muestras de la ciudad de Rio Branco. Finalmente, el trabajo concluye que: i) el tamaño promedio de grano de los sedimentos del fondo del río Acre, disminuirá en diámetro a lo largo de la ruta de Assis Brasil a Río Branco; ii) la técnica de morfometría de sedimentos mostró evidencia del proceso de desgaste y removilización de las partículas desde la salida de la roca matriz hasta su deposición; iii) el uso del software ImageJ demostró ser eficiente en velocidad y precisión para el análisis y la diferenciación morfométrica de sedimentos arenosos en la sección analizada.
\end{abstract}

Palabras-clave: Dinámica del río; Granulometría de sedimentos; Morfometria; Rio Acre; Sedimentología. 


\section{INTRODUÇÃO}

Por sedimentos compreende-se todo material proveniente da degradação de rochas préexistentes, as quais estão susceptíveis ao transporte e deposição. A sua classificação decorre das características físicas: ambiente deposicional, ou conforme o tipo de sedimentação. (GUERRA; GUERRA, 2006).

O estudo dessas partículas teve seu início em meados do século XX, quando Pentland (1927), Wadell (1932), Riley (1941), e alguns outros autores desenvolveram técnicas particulares, objetivando definir sua forma.

Nesse sentido, a forma dessas partículas é uma propriedade importante, pois o comportamento hidrodinâmico está intrinsicamente relacionado com a forma. Desse modo, as partículas mais angulosas detêm de maior dificuldade no processo de remobilização do que as mais esféricas (DIAS, 2004).

O estudo morfológico é relevante, visto que, leva a uma melhor compressão dos fatores externos a forma, tais como: as características da rocha matriz, a distância a qual foi submetida até o ambiente deposicional, agentes envolvidos no transporte, a durabilidade e resistência a abrasão e as zonas de fraturas e diacláses presentes nas partículas (DIAS, 2004; RIBEIRO; BONETTI, 2013).

A descrição da forma de partícula pode ser classificada como qualitativa ou quantitativa. Qualitativo descreve, em termos de palavras, a forma da partícula (por exemplo, alongada, esférica, escamosa, etc.) e quantitativo que relaciona as dimensões medidas no campo da engenharia. Sendo assim, a descrição quantitativa da partícula torna-se mais importante devido à sua reprodutibilidade (RODRIGUEZ et.al., 2013).

Métodos eficazes são bem desenvolvidos e aceitos para análise de partículas maiores como areia e cascalhos. Em mensurações dos grãos no tamanho areia é comum a utilização de estereoscópicos, microscópio óptico ou lupa binocular com câmera fotográfica acoplada, a fim de quantificar: a área, perímetro e volume, possibilitando as bases para análise dos descritores de forma como: circularidade, índice de alongamento, diâmetro, dentre outros (RIBEIRO; BONETTI, 2013; TEIXEIRA et. al., 2017; SERRANO et al., 2019).

Suguio (2003), ressalta que os métodos de medida da morfometria sedimentar devem ser aplicados conforme a distribuição granulométrica, uma vez que, os significados de forma como esfericidade e arrendamento, diferenciam-se para os grãos mais grossos como o cascalho, dos mais finos como as areias. 
É importante salientar que com o desenvolvimento tecnológico, os estudos sedimentológicos ganharam um importante aliado nos descritores de forma. A análise de imagens baseadas em computador foi aplicada na pesquisa de peneiramento, trazendo novos métodos práticos para determinar o tamanho de partícula com bons resultados práticos (ANDERSSON, 2010; SERRANO et al., 2019).

Trabalhos como o de Viana, et. al., (2016), é de suma importância, pois apresenta um guia contendo os passos e os resultados do teste de um método para a quantificação de imagens digitais de amostras de areias, baseado no uso de imagens digitais microscópicas processadas no programa ImageJ.

No trabalho de Serrano et. al., (2019), é descrita uma metodologia para análise de morfometria de sedimentos de fundo com aplicação no rio Acre, obtendo resultados significativos como: a fração de areia foi maior na margem esquerda dos pontos observados, característico de regiões com perda de material sedimentar, e o software ImageJ 1.52t mostrouse apropriado para determinação dos padrões morfométricos dos grãos de sedimentos, permitindo a padronização dos atributos morfométricos.

Para o presente trabalho, também foi adotado o software livre (ImageJ) desenvolvido por Rasband (2004). Assim, os objetivos do artigo são: (i) determinar o perfil de forma dos grãos dos sedimentos de fundo; (ii) avaliar a aplicação de técnicas de processamento de imagem associadas ao cálculo de índices morfométricos, adjunto da estatística descritiva para quantificar a variabilidade dimensional destes sedimentos; (iii) fazer um comparativo entre as diferentes frações granulométricas encontradas nos pontos de coleta, nas cidades de Assis Brasil, Brasiléia e Rio Branco.

\section{MATERIAL E MÉTODOS}

Inicialmente, foi analisada a variação granulométrica dos sedimentos do rio Acre entre as cidades de Assis Brasil, Brasiléia e Rio Branco para identificar se o processo de transporte ao longo do trajeto proporcionou a redução do tamanho dos grãos de sedimento (Figura 1). Em seguida, analisou-se a morfometria de sedimentos das amostras da cidade de Rio Branco, para descrever forma dos grãos.

Para a análise dos sedimentos no trecho da cidade de Rio Branco, utilizou-se seis amostras provenientes do leito do rio Acre, coletadas na margem esquerda e direita (Figura 1). Em laboratório iniciou-se a análise granulométrica com o propósito de quantificar o percentual 
de areia, silte e argila, conforme metodologia descrita por (ABNT-NBR 7181, 1984; MIRRA et.al., 2014).

Figura 1: localização dos pontos de coleta - Rio Acre. Elaborado por: LUCIO; SERRANO, 2020.

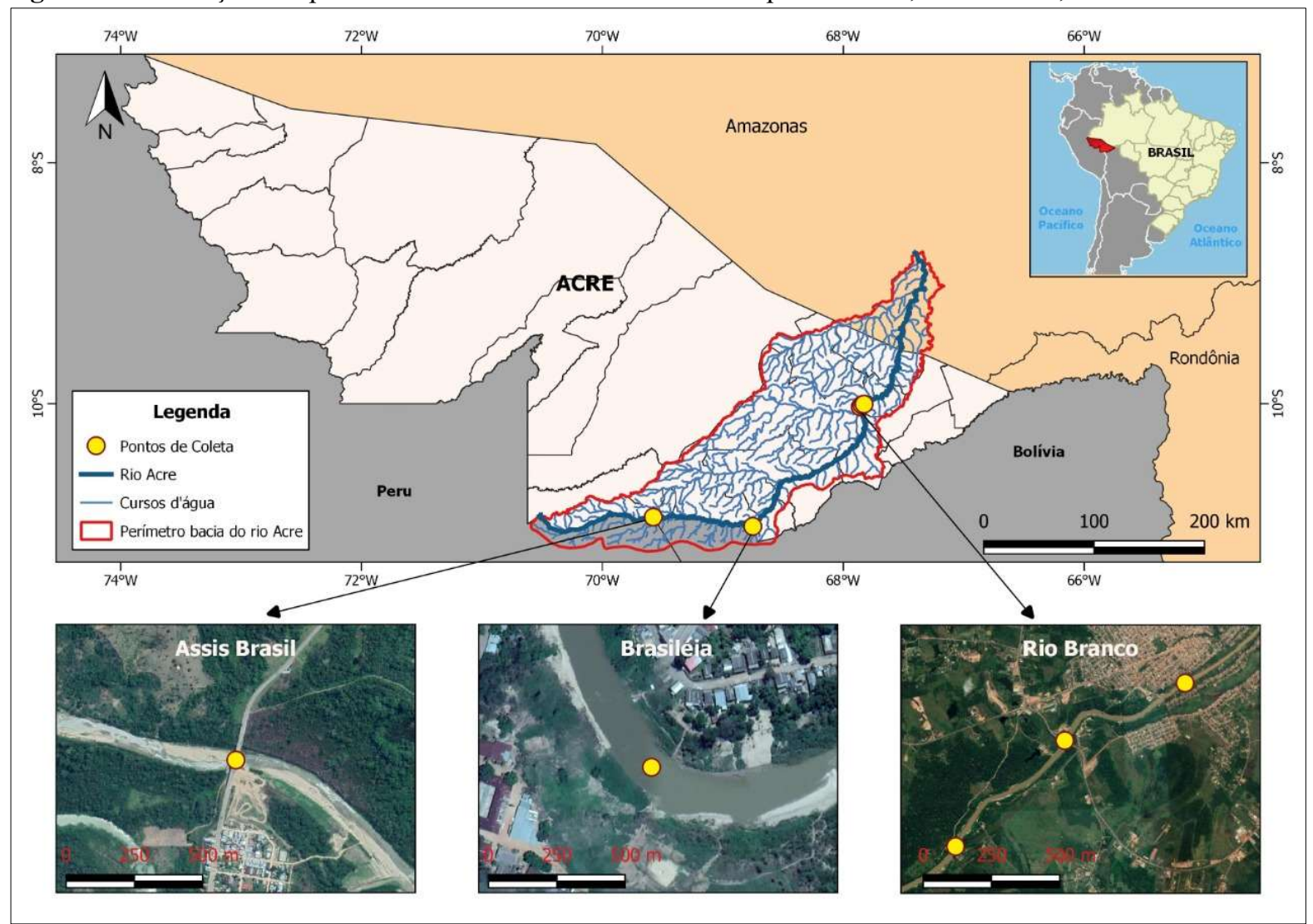

Para a observação das variáveis morfológicas das partículas, adotou-se o uso do software ImageJ 1.52t (RASBAND, 2004). De modo que, a descrição se resume em três etapas principais e uma complementar:

\section{$1^{\circ}$ Etapa: análise da variação granulométrica das amostras das cidades de Assis Brasil, Brasiléia e Rio Branco.}

- Amostra para análise granulométrica (peneiramento grosso)

Conforme as Normas Brasileiras (NBR-6508, NBR-6457 e NBR-7181), trabalhou-se com amostras representativas de $120 \mathrm{~g}$, obtida através do repartidor de amostras ou quarteamento, pretendendo obter amostra suficiente para realização dos ensaios requeridos.

Uma vez destorroadas as amostras, todo o material foi distribuído na peneira de tamanho 2,0 mm. A massa retida foi então lavada na própria peneira. Assim, todo material fino silte e argila presente na amostra formão retirados. 


\section{- Amostra para análise granulométrica (peneiramento fino):}

Após a lavagem, todo o material retido foi identificado e levado à estufa para secagem, durante um período de 24 horas. Procedeu-se então com o peneiramento do material seco nas peneiras 1,$19 ; 0,59 ; 0,42 ; 0,25 ; 0,15 ; 0,09 ;$ e $0,0106 \mathrm{~mm}$ de diâmetro, mediante agitador mecânico por cerca de 10 minutos a média rotação. No fim, todas as frações retidas em cada peneira foram pesadas, para o cálculo de porcentagem do total retido.

\section{$2^{\circ}$ Etapa: preparação e obtenção das imagens dos sedimentos da cidade de Rio Branco:}

\section{- Amostra para análise morfométrica:}

No caso da análise morfométrica, selecionou-se um conjunto de grãos ao acaso da fração areia de cada uma das amostras retidas em peneira com malha $250 \mu \mathrm{m}$, referentes ao município de Rio Branco, levando em consideração as margens, onde realizaram-se as coletas.

Para cada amostra, capturou-se de forma aleatória três imagens, visando selecionar as de melhor qualidade e menor contato entre as partículas na lâmina, evitando interferências na posterior quantificação. Todas as fotografias obtidas, forma realizadas no estéreomicroscópio, modelo Leica M205A, sobre fundo em cor preto, com magnificação de 20.5x e zoom ótico na fotografia de 100x e salvas em formato comprimido do tipo "TIFF" (RIBEIRO; BONETTI, 2013; VIANA et al., 2016).

\section{- Tratamento e quantificação digital das imagens}

Uma vez obtidas todas as imagens, realizou-se o processamento digital, por meio do software livre ImageJ (Rasband, 2004), disponível para download de forma gratuita, muito utilizado na comunidade científica devido à grande quantidade de complementos (plugins) desenvolvidos que melhoram as análises pertinentes ao objetivo de estudo de cada pesquisador.

Primeiramente, todas as imagens originais coloridas foram previamente taradas, convertidas em formato binário (preto e branco, 8-bits), intercalando 256 níveis de cinza, para que, fosse possível o reconhecimento o algoritmo do software na mensuração e cálculo das feições descritas. Este é um passo fundamental, pois propicia a correta identificação das partículas medidas, sendo um dos pontos chave para o sucesso da técnica (VIANA et al., 2016; TEIXEIRA et. al., 2017). 
O procedimento adotado utilizado na quantificação das areias, foi adaptado da metodologia descrita por Viana et. al., (2016), "binarização via filtro de limiar". Essa metodologia é mais prática e útil, permitindo a conversão das imagens em formato binário. Desse modo, os procedimentos foram executados da seguinte forma:

a) Conversão da imagem para formato binário: Image $>$ Type $>8$-Bit.

b) Calibragem das imagens mensuradas: consistiu na calibragem de todas as imagens em lâminas graduadas, previamente taradas na escala da imagem. Através da ferramenta de desenho de linha reta (*Straight*), definimos uma distância conhecida $(200 \mu \mathrm{m})$, para que, o software pudesse reconhecer e fazer as mensurações em: Analyse $>$ Set scale. Posteriormente, a distância conhecida de $200 \mu \mathrm{m}$ foi indexada em ("Known distance"), e a unidade de medida é identificada em: (“Unit of lenght”). Para manter a formatação e calibragem de todas as imagens, marcou-se a caixa "Global”, aplicando “ok”. Desse modo, todas a imagens posteriores manteriam a distância conhecida na mensuração.

c) Conversão das imagens em formato binário (preto e branco), pós-calibragem: após a calibragem, ocorreu a conversão de todas as imagens para o formato binário da seguinte forma: Process $>$ Binary $>$ MakeBinary. Após as transformações, nos casos de ocorrência de ruído decorrente da reflexão desigual do quartzo, realizou-se a correção, descrita a seguir.

d) Correção de ruídos: Para a correção dos ruídos, utilizou-se os plugins descritos por Ribeiro e Bonetti (2013), desenvolvidos por Landini (2006) incluídos ao pacote Morphological Operators for ImageJ, disponível para download gratuito na plataforma de plugins do próprio software. Dentre eles: BinaryFill_2; BinaryFilterReconstruct_.class e BinaryKillBorders_class. Para os demais problemas remanescentes, retificamos com auxílio das ferramentas de pintura ("PaintBrushToll e FloodFillTool”), inseridas no próprio ImageJ. Cabe ainda mencionar que para os objetos incompletos (próximos as bordas, ou adjunto de outra partícula), descartamos da análise, a fim de minimizar os erros.

e) Procedimento de análise: em: Analyse $>$ Analyse particles, digita-se a área mínima de 0,01 pixel, tendo em vista, que outros pequenos ruídos remanescentes na imagem pudessem ser mensurados. Marcou-se então, o campo de excluir bordas (“Exclude edges”) aplicado “ok”.

f) Salvar os resultados: na nova janela "Results", salvou-se os arquivos em: File $>$ save as, definindo o diretório e nome do arquivo a ser gravado. 


\section{- Atributos morfométricos utilizados na análise:}

Área: compreende-se o número de pixels presente no polígono. Uma vez calibrada, a área é mensurada na unidade a qual foi calibrada, caso contrário, apresentará o valor em pixels (VIANA, et.al., 2016).

Perímetro: é definido pelo comprimento do lado externo do objeto aqui, dos grãos de areia mensurados. O perímetro é computado conforme a calibração, não havendo a mesma, o valor é apresentado em pixels (VIANA, et.al., 2016).

Arredondamento: não pode ser confundido com a esfericidade, portanto Ribeiro e Bonetti (2013, p. 38), definem como sendo: “[...] expressão dos graus de curvaturas das arestas e vértices do contorno das partículas [...]”. De modo que, seu resultado varia entre 0 a 1 . Quanto mais próximo de 1 , mais arredondado é o objeto. Se o valor apresentado for 1, pode-se inferir que o objeto é um círculo perfeito (VIANA, et.al., 2016). Cox (1929) apud Ribeiro e Bonetti (2013), calculou o arredondamento de partículas, baseando-se no comparativo da área em função do perímetro, através da seguinte equação:

$A r=\frac{4 \pi A}{P^{2}}$

Onde:

$A r=$ Arredondamento (adimensional).

$A=$ área do objeto $\left(\mu \mathrm{m}^{2}\right)$.

$P=$ perímetro do objeto $(\mu \mathrm{m})$.

Alongamento: é o resultado obtido pela divisão do menor eixo, pelo maior eixo. A partir de então, o valor obtido deve variar entre 0 e 1 . Se o valor for igual a 1 , o objeto é aproximadamente circular ou quadrado. Na medida que se afasta de 1, o objeto torna-se mais alongado (VIANA, et.al., 2016). Desse modo, pode ser calculado através da seguinte expressão:

$$
\mathrm{AL}=\frac{\mathrm{Me}}{M a}
$$

Onde:

$\mathrm{AL}=$ Alongamento (adimensional).

$\mathrm{Me}=$ menor eixo $(\mu \mathrm{m})$.

$\mathrm{Ma}=$ maior eixo $(\mu \mathrm{m})$.

Compacidade: muito importante, pois é responsável por viabilizar a medida da circularidade do objeto. Seu valor também se situa entre 0 e 1 . Se o valor aprestando for 1 a 
partícula é aproximadamente circular. Vale também ressaltar, a correlação que há com o alongamento da partícula; quanto maior for o alongamento da partícula, menor será seu eixo principal, como resultado menor será a circularidade (RIBEIRO; BONETTI, 2013). Para o cálculo, tem-se a equação:

Co: $\frac{\frac{\sqrt{4 \mathrm{~A}}}{\mathrm{MA}}}{\mathrm{MA}}$

Onde:

$\mathrm{Co}=$ compacidade (adimensional).

$\mathrm{A}=$ área $\left(\mu \mathrm{m}^{2}\right)$.

$\mathrm{Ma}=$ maior eixo $(\mu \mathrm{m})$.

\section{RESULTADOS E DISCUSSÃO}

\subsection{Fração granulométrica}

Os dados demonstram que as partículas de maior tamanho provêm do município de Assis Brasil, seguidos de Brasiléia e por fim Rio Branco. Uma vez no ciclo sedimentar estes elementos estão sujeitos ao transporte em diferente intensidade. Assis Brasil, por se situar mais a montante do que os demais municípios, detém de um fluxo de energia maior, uma vez que, as partículas se desgastam gradativamente ao serem transportadas no canal fluvial, consequentemente apresentam diâmetro maior do que as encontradas em Brasiléia e principalmente em Rio Branco, ocasionadas pelo desgaste no processo de remobilização (Figura 2). 
Figura 2: Curva granulométrica referente as amostras coletadas em Assis Brasil, Brasiléia e Rio Branco.

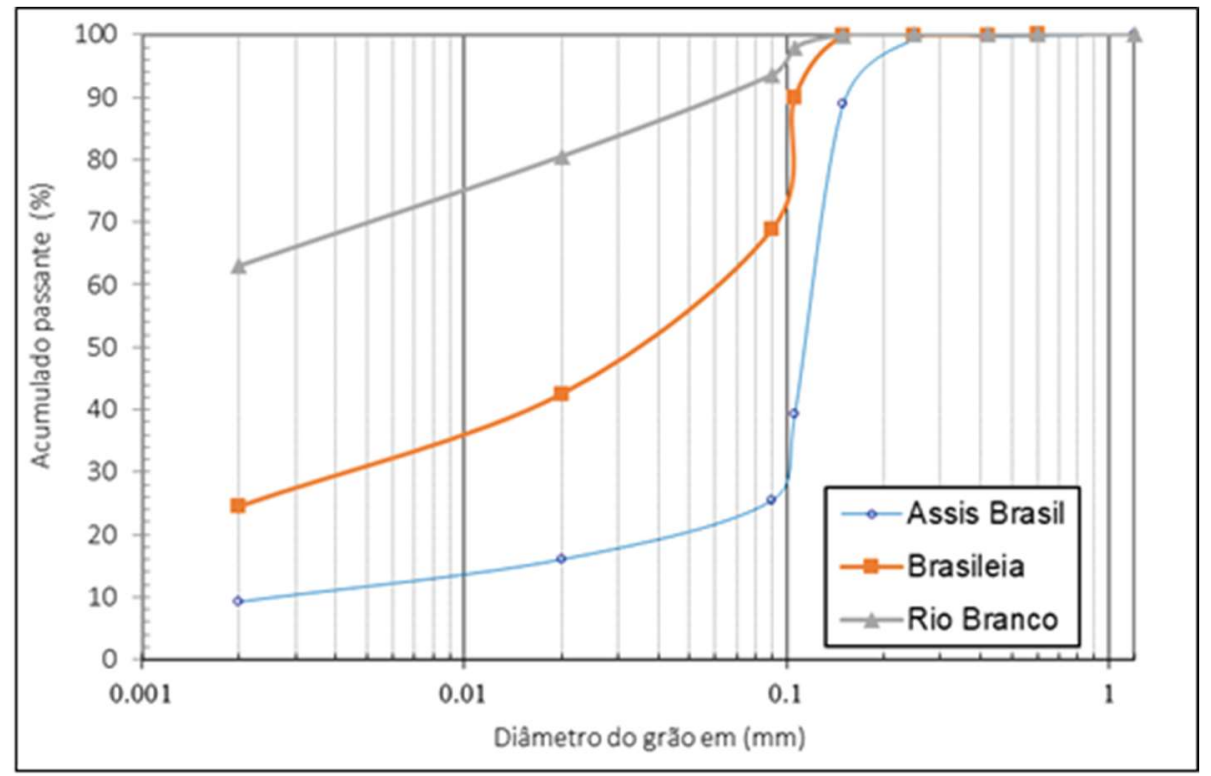

Já no tocante das areias obtidas para a quantificação e descrição da forma dos grãos, foram separadas através do processo de peneiramento e método da pipetagem descrito por (MIRRA et.al., 2014). Nesse sentido, observa-se que há uma predominância de areia e silte em $50 \%$ das amostras. O que dá um indicativo que na predominância de areia, o fluxo tende a ser maior, pois as partículas de granulometria reduzida (silte e argila), tendem a manter-se em suspensão e seguir o fluxo enquanto este for suficiente para mantê-las (CHRISTOFOLETTI, 1980).

Outra observação importante que pode influenciar nos valores é a extração de areia do leito fluvial (dragagem) descrita por Accorsi (2014), onde ocorrem principalmente a montante dos pontos de coleta, podendo influenciar na dispersão e consequentemente deposição, do material não aproveitado pelas dragas, que retorna ao leito fluvial (Tabela 1).

Tabela 1 - Fração granulométrica das amostras - rio Acre (Rio Branco). Elaborado pelos autores.

\begin{tabular}{ccccc}
\hline Pontos & Areia total (\%) & Argila total (\%) & Silte total (\%) & Total \% \\
\hline PE5 & 68,38 & 6,31 & 25,30 & 100 \\
\hline PD5 & 37,65 & 17,04 & 45,31 & 100 \\
\hline PE6 & 61,34 & 8,07 & 30,59 & 100 \\
\hline PD6 & 47,37 & 5,78 & 46,85 & 100 \\
\hline PE7 & 34,39 & 7,03 & 58,58 & 100 \\
\hline PD7 & 20,69 & 6,02 & 73,28 & 100 \\
\hline
\end{tabular}




\subsection{Análise descritiva (amostras de Rio Branco)}

\subsubsection{Diâmetro dos grãos}

Ao término do processamento e quantificação dos resultados via software BioEstat 5.0, pode-se observar que o diâmetro médio dos grãos variou entre $(68,71 \mu \mathrm{m}$ e $94,69 \mu \mathrm{m})$, apresentando os menores resultados nas margens direita dos pontos 5 e 7. Para a mediana, esta que divide o diâmetro entre duas metades, o menor valor apresentado decorreu na margem direita do ponto 5 , cujo valor obtido foi de $(73,34 \mu \mathrm{m})$, também a maior foi registrada no referido ponto, porém na margem esquerda com $(91,61 \mu \mathrm{m})$.

Objetivando o maior aprofundamento na análise e atenuações de erros, a média geométrica apresentou valores situados entre $(67,20 \mu \mathrm{m}$ e $91,89 \mu \mathrm{m})$, novamente os menores valores obtidos foram na margem direita nos pontos 5 e 7, o que pode ser explicado pelo ambiente deposicional a qual estão inseridos. Quanto maior o fluxo do canal fluvial, menos tempo as partículas ficam depositadas no leito, e consequentemente as de menor diâmetro tendem a ser transportadas com maior facilidade.

$\mathrm{Na}$ variabilidade, as maiores amplitudes foram registradas no ponto 6 , tanto para a margem direita quanto esquerda $(80,40 \mu \mathrm{m}$ e $82,05 \mu \mathrm{m})$ respetivamente, o que pode ser explicado pelo tamanho médio dos diâmetros conforme (figura 3). As maiores variâncias e desvio padrão entre os pontos, também decorrem de valores apresentados pelo ponto 6 em ambas as margens, com $(625.40 \mu \mathrm{m}$ e $700.02 \mu \mathrm{m})$, para a variância e $(25.08 \mu \mathrm{m}$ e $26.45 \mu \mathrm{m})$ equivalente ao desvio padrão.

Figura 3: diâmetro médio dos grãos pontos (5, 6 e 7) - Rio Acre. Elaborado pelos autores.

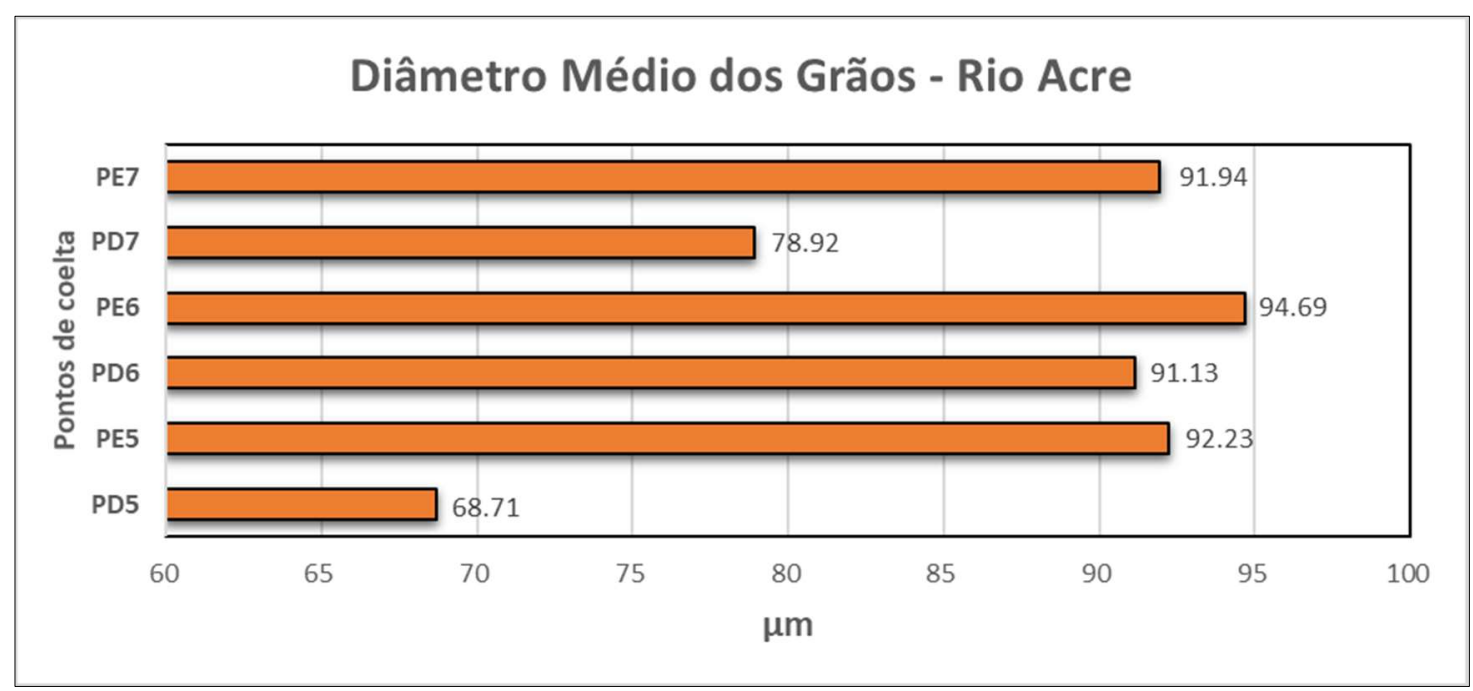




\subsubsection{Arredondamento}

O arredondamento diz respeito a curvatura ou angulosidade das arestas e vértices da partícula. Desse modo, torna-se importante sua análise, pois é um indicativo do tempo em que os sedimentos se encontram em atividade no ciclo sedimentar, intensidade de transporte e distância do lócus de origem dessas partículas (DIAS, 2004).

Ao analisar a tendência central, as medianas pouco variaram, pois apresentam resultados situados entre $(0,68)$ ponto 6 (margem direita), e $(0,77)$ em ambas as margens do ponto 5 . Adjunto da média aritmética, com os maiores valores obtidos na margem esquerda dos pontos 5 e $6(0,76$ e 0,75$)$ respectivamente, e média geométrica que também indicou maior valor situado na margem esquerda dos pontos 5 e $6(0,76$ e 0,74$)$. Nesse sentido, há um indicativo de que nesses locais em específico, as partículas tendem a ser menos angulosas, devido ao desgaste proveniente do ciclo sedimentar e distância, bem como, apresentam maior facilidade a serem remobilizadas.

Figura 4: arredondamento médio dos grãos - Rio Acre. Elaborado pelos autores.

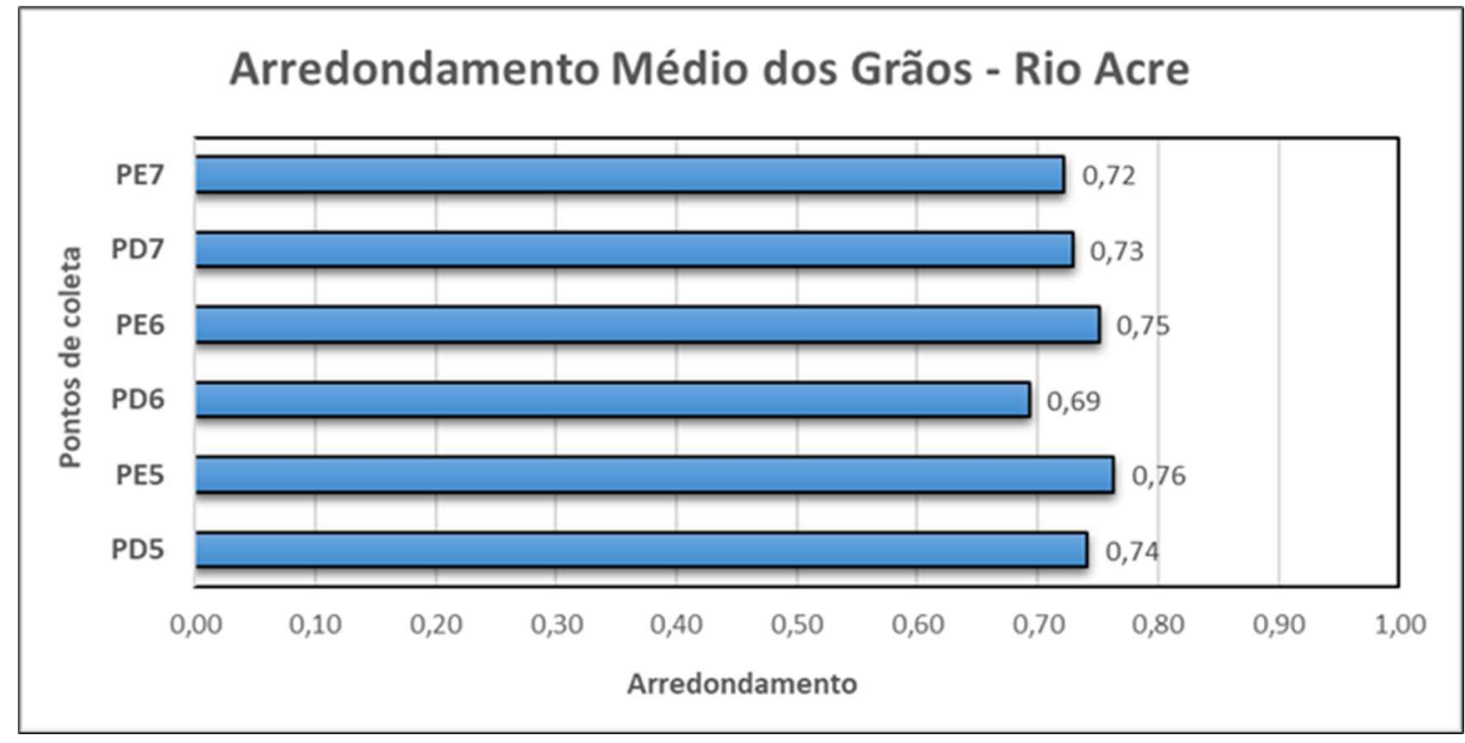

Na variância e desvio padrão tivemos valores bem pequenos, decorrente a proximidade que eles apresentaram, alternando assim, entre $(0,002$ e 0,005$)$ variância e $(0,048$ a 0,073). Para a amplitude, os maiores valores obtidos na margem esquerda dos pontos 6 e 7 com $(0,24$ a 0,22). 


\subsubsection{Compacidade}

A compacidade "circularidade", diz respeito, a proximidade que a partícula tem de se aproximar de uma forma esférica. Nesse sentido, as medianas situam-se entre $(0,45$ a 0,52$)$ valores obtidos na margem direita e esquerda do ponto 6 , as quais os demais encontram-se inseridos nesse intervalo (figura 5).

Figura 5: compacidade média dos grãos - Rio Acre. Elaborado pelos autores.

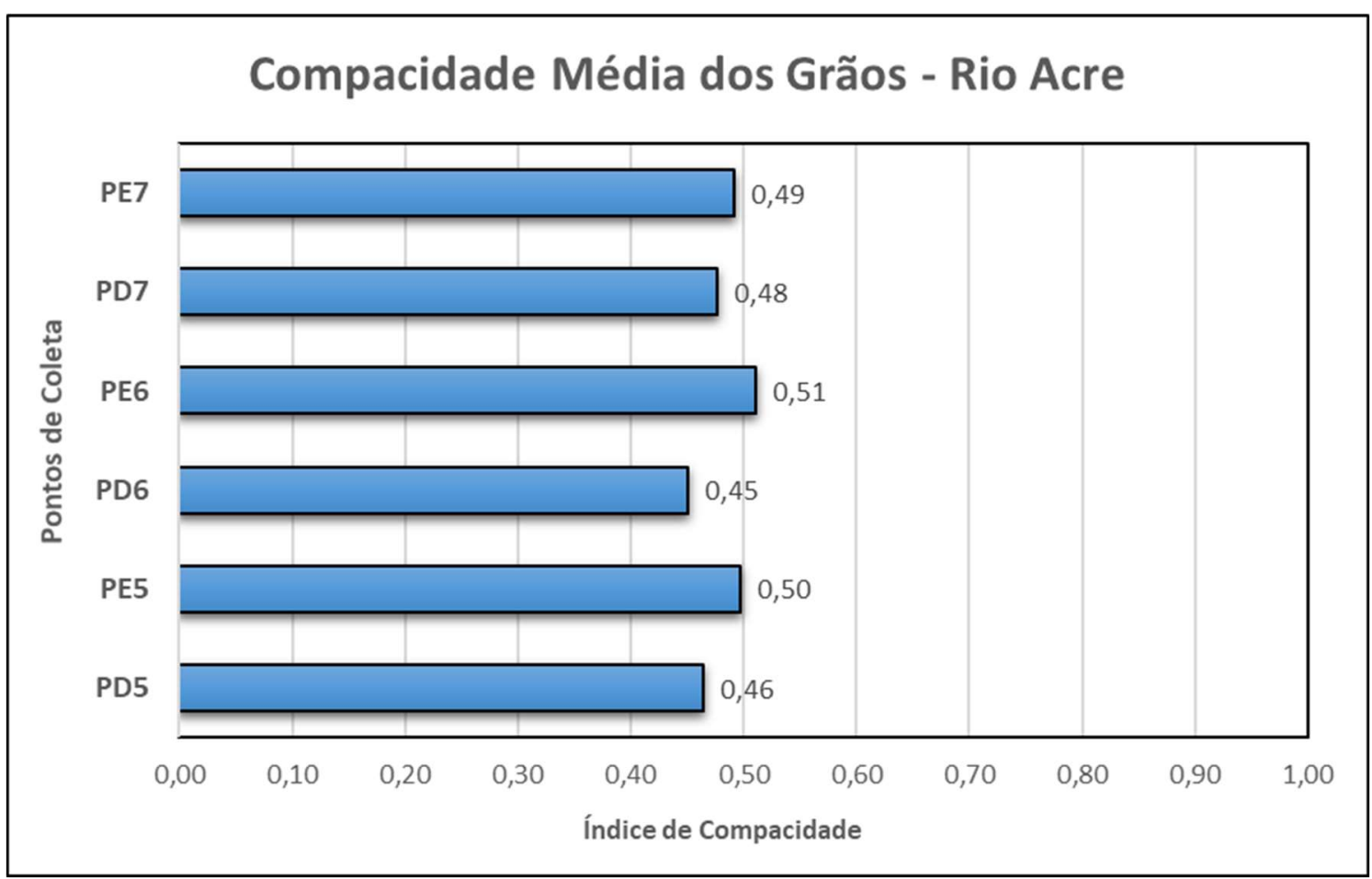

As maiores médias aritméticas registradas, foram na margem esquerda dos pontos $5 \mathrm{e}$ 6, consequentemente também a média geométrica nesses dois pontos, dispôs-se em números próximos a $(0,49$ e 0,51$)$. Desse modo, a partir das médias, pode-se inferir que as partículas ainda estão em processo de desgaste decorrente da longa distância do ponto de origem e o ciclo sedimentar.

O desvio padrão referente aos valores do ponto 6, apresentou decimais próximos entre (0.024 e 0.046), combinada com uma baixa variância que apenas na margem direita do ponto 6 apresentou valor de $(0,002)$ e os demais pontos e margens apresentaram valor próximo a $(0,001)$. 


\subsubsection{Alongamento}

Menos usual que os demais índices supracitados, indica se o objeto apresenta uma forma próxima circular ou quadrada. Desse modo, as medianas situaram-se em valores (0.65 e 0.84), sendo que as de maior valor concentram-se na margem esquerda dos três pontos. As maiores médias aritméticas, foram registradas na margem esquerda dos três pontos (figura 6), o que pode ser confirmado também com os valores obtidos pela média geométrica $(0,77 ; 0,81$ e 0,75$)$ respectivamente. Portanto, devido aos valores estarem em proximidade a $(1,0)$, tais partículas novamente, apresentam-se com características próximas circulares, o que indica desgaste no processo de remobilização, bem como, elevada distância entre o ponto de origem ao ambiente deposicional a qual estão inseridas.

Figura 6: alongamento médios dos grãos - Rio Acre. Elaborado pelos autores.

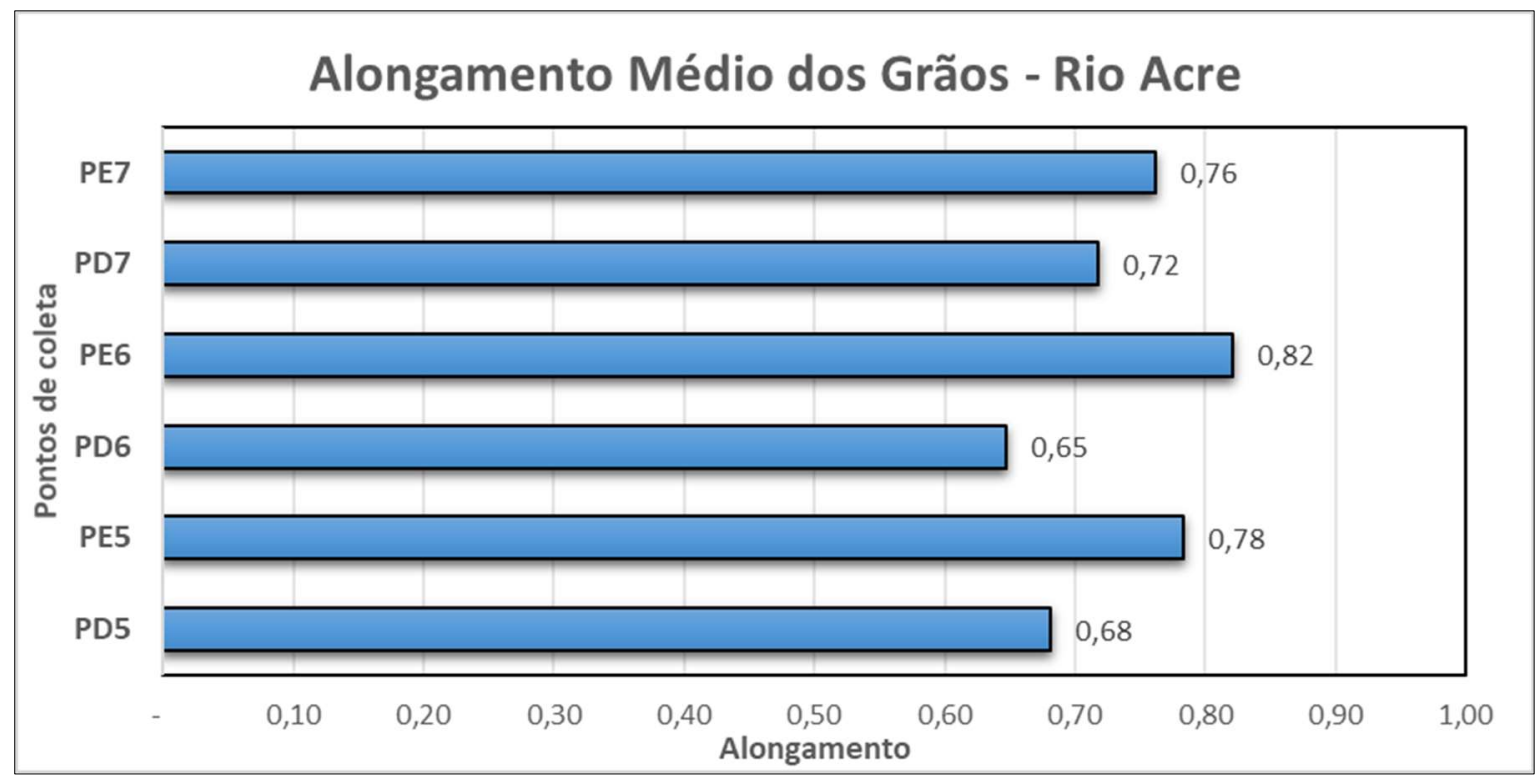

No tocante a variabilidade do alongamento, os dados obtidos indicam que a variância se encontra bem próxima da média por apresentar valores próximos variando entre $(0,008$ a 0,016$)$, concomitante a amplitude de maior expressão para a margem esquerda do ponto $6(0,43)$ e (0.40) para a margem esquerda do ponto 7. Por fim, o desvio padrão apresentou alternância entre as margens, sendo que também os maiores valores obtidos foram na margem direita do ponto $6(0.12)$ e margem esquerda do ponto 7 (0.11), evidencia-se assim, a proximidade dos valores, a média indicando proximidade a circularidade descrita nos itens anteriores. 


\subsection{Descritor de forma das amostras}

Para definir a morfologia dos grãos, optou-se em primeiro momento realizar a descrição conforme escala proposta por Powers (1953), pois esta permite uma avaliação comparativa bidimensional, classificando os grãos de acordo com sua representação na imagem (DIAS, 2004). Nesse sentido, as classes definidas por Powers (1953) apud Dias (2004) foram: muito angulares; angular; sub-angular; sub-rolado; rolado; e bem rolado (figura 7).

Figura 7: exemplos das 6 classes de rolamento definidas por Powers. a - muito angular; $\mathrm{b}$ - angular; c - sub- angular; d - sub-rolado; e - rolado; f - bem rolado. Adaptado de Dias (2004).

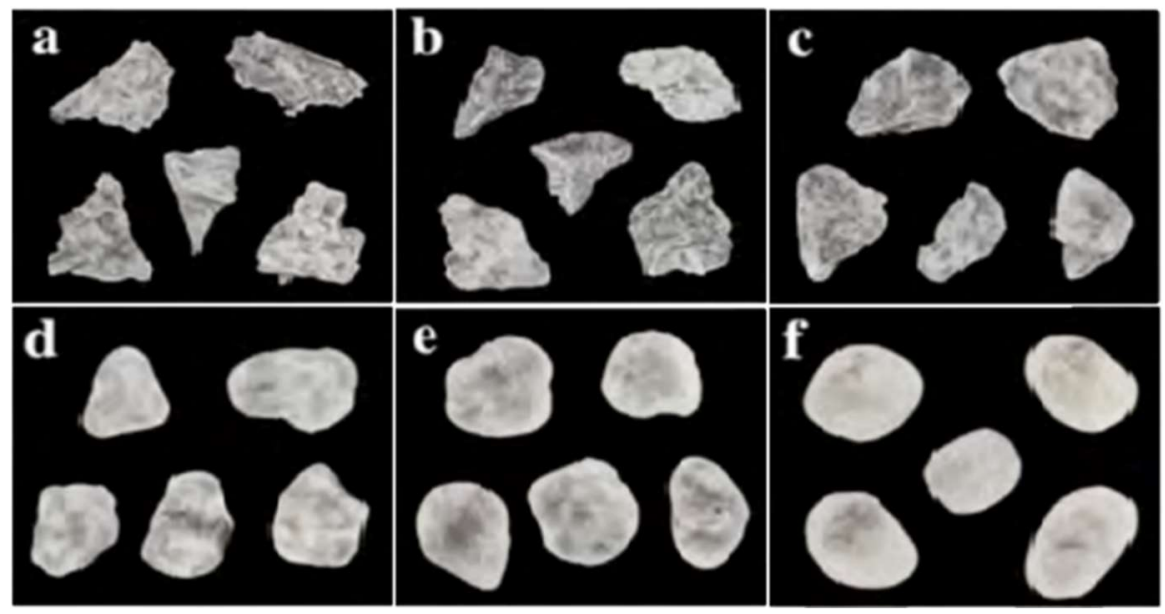

As amostras obtidas no ponto 5 (margem direita) podem ser classificas como: sub- angular a sub-rolado. Para a margem esquerda, podem ser classificados como: subangular, sub-rolado e rolado conforme ilustrado na (figura 8. A e B.).

Figura 8: A. Morfologia dos grãos processados ponto 5 (margem direita). B. Morfologia dos grãos processados ponto 5 (margem esquerda). Adaptado de: SERRANO, et. al., 2019.

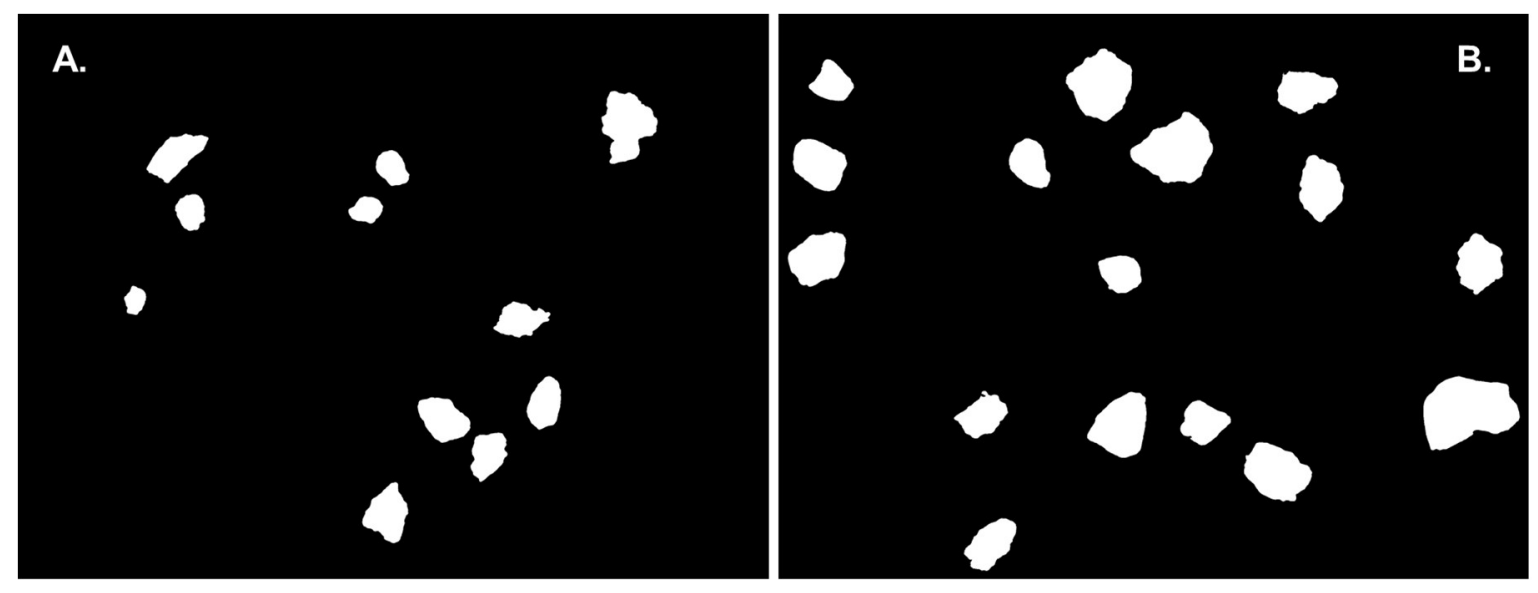


Ao ponto 6 (margem direita) os grãos foram classificados como: sub-angular a subrolado. Já para a margem esquerda tivemos a presença de partículas: sub-angular; sub-rolado; rolado (figura 9. A e B.).

Figura 9: A. Morfologia dos grãos processados ponto - 6 (margem direita). B. Morfologia dos grãos processados ponto 6 (margem esquerda). Adaptado de: SERRANO, et. al., 2019.
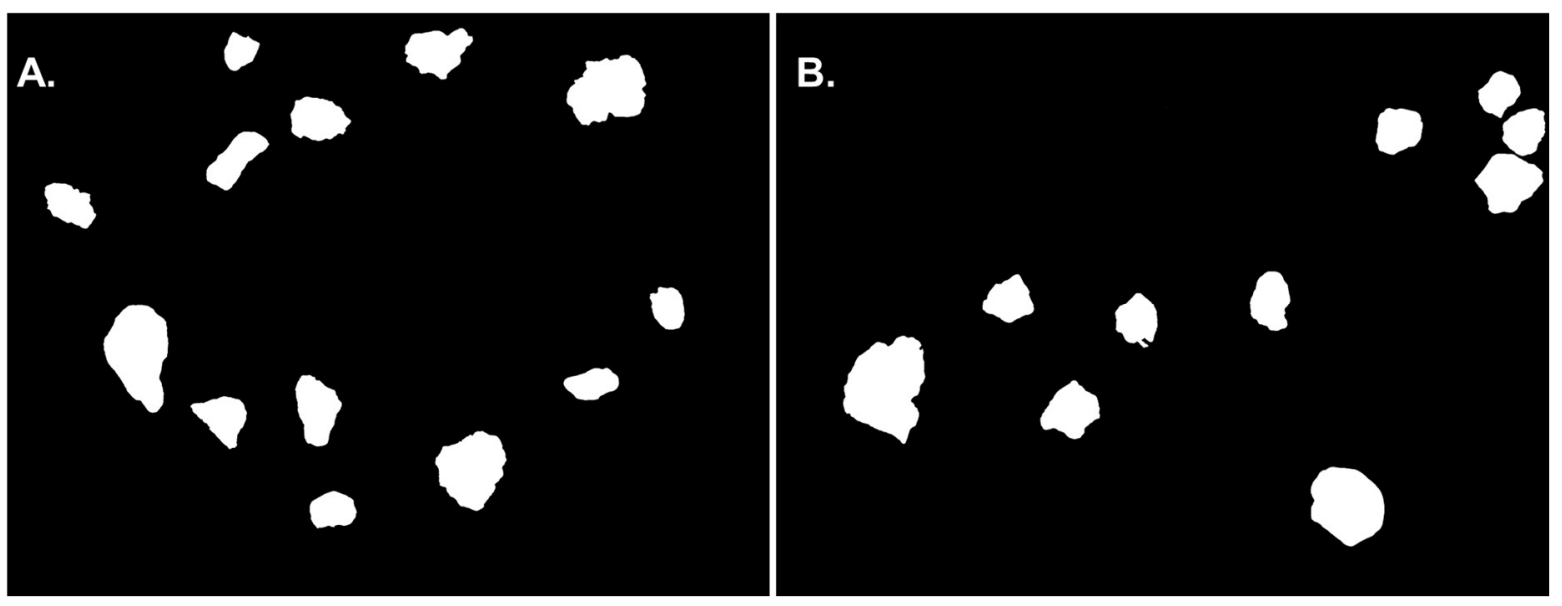

Por fim, ao ponto 7 (margem direita) a forma dos grãos indicou a classificação em: angular e sub- angular. A margem direita apresentou forma: sub-angular a sub-rolado, conforme ilustrado (figura 10. A e B.).

Figura 10: A. Morfologia dos grãos processados ponto 7 (margem direita). B. Morfologia dos grãos processados ponto 7 (margem esquerda). Adaptado de: SERRANO, et. al., 2019.
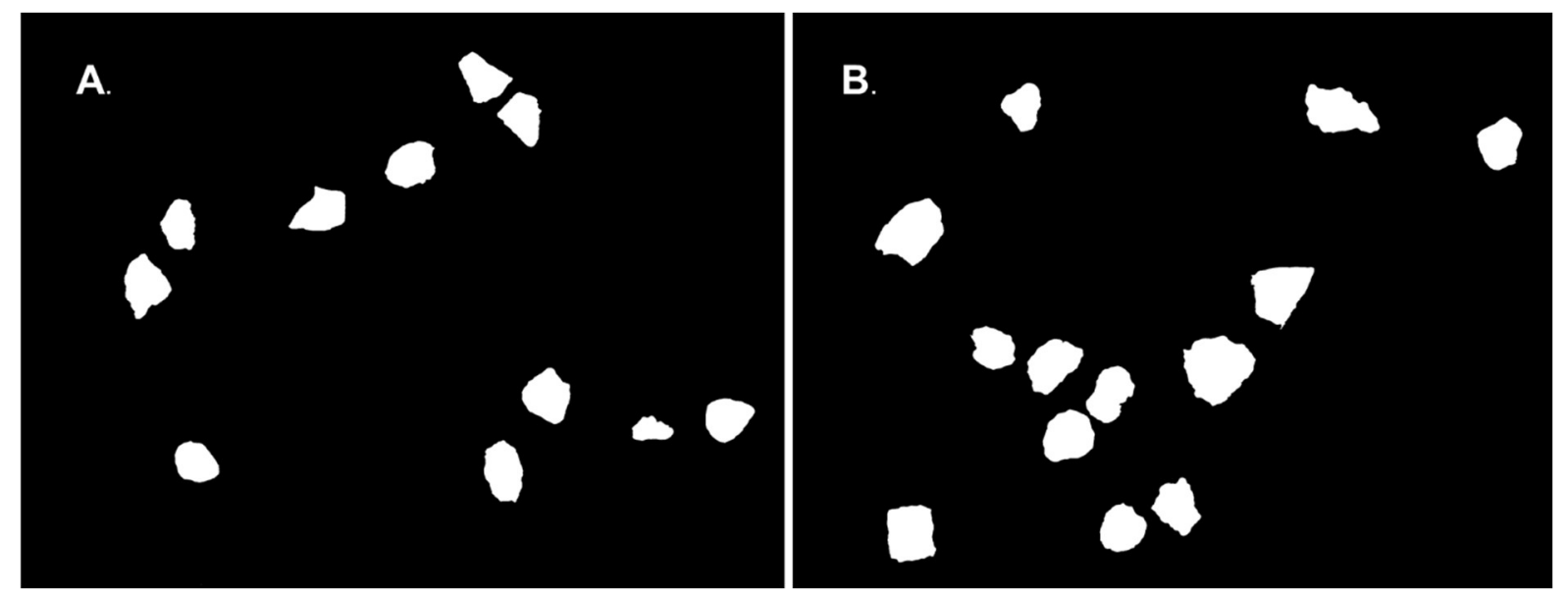

A segunda etapa, consiste na análise dos índices presentes na tabela descrita por Cox (1927), baseada no índice de arredondamento, apresenta as seguintes classes: muito angular $(0,50-0,65)$; angular $(0,65-0,70)$; sub-angular $(0,70-0,75)$; sub-arredondado $(0,75-0,77)$; arredondado $(0,77-0,80)$ e bem arredondado $(0,80-1,00)$. Nesse sentido, de acordo com a tabela proposta as amostras podem ser classificadas da seguinte forma: sub angular para ponto 
5 (margem direita), por apresentar valor médio (0.74). A margem esquerda: arredondado, pois apresentou valor médio de $(0,76)$. No ponto 6 (margem direita): angular com valor médio de $(0,69)$ e sub-angular para (margem esquerda), que apresentou valor médio de $(0.75)$. Por fim, sub-angular ao ponto 7 que (em ambas as margens) as médias obtidas foram (0.72), semelhante as classificações realizadas por Serrano et al. (2018), quando avaliou os desgastes causador pelos sedimentos em rotores de bombas.

Dias (2004), ainda complementa afirmando, que além da imagem é necessário considerar as mineralogias dos grãos, pois os mais resistentes tentem a ser rolados em menor velocidade, do que os de menor resistência. Desse modo, as amostras ao variar entre: a angular; c - sub-angular; $\mathrm{d}$ - sub-rolado; $\mathrm{e}$ - rolado indicam que boa parte do material apresenta uma elevada durabilidade característica de uma estrutura sedimentar ainda recente.

\section{CONCLUSÃO}

Diante do exposto, o presente estudo demostra que a granulometria dos sedimentos do rio Acre, apresentam a redução do seu diâmetro médio ao longo de curso em um trecho de aproximadamente $400 \mathrm{~km}$, sugerindo que durante seu transporte os sedimentos vão sendo retrabalhados, perdendo parte de sua massa.

A utilização de microfotografias e classificação pelo software ImageJ, para análise dos descritores de forma, permitiu uma análise rápida e padronizada dos atributos morfológicos dos grãos de sedimentos. Os resultados obtidos através dos atributos morfométricos adotados no presente estudo, puderam evidenciar uma compressão dos processos de desgaste decorrente da remobilização nas partículas durante o processo de transporte.

O material situado mais a montante, apresentam maior diâmetro, angulosidade e tamanho, já as situadas mais a jusante, devido ao desgaste ocasionado pelas sinergias do sistema, tendem a ser menos angulosas, de menor diâmetro e mais circulares, consequentemente exprimem facilidade de seguir no curso do rio.

Por fim, é importante salientar, que a metodologia passa por processo de adaptação e por isso, propõe-se a elaboração de estudos posteriores de maior aprofundamento, a fim de fazer o comparativo propondo maior conhecimento da dinâmica do ciclo sedimentar do rio Acre.

\section{AGRADECIMENTOS}

Agradecimento especial à Universidade Federal do Acre por disponibilizar os meios para elaboração do estudo, como também, aos professores e demais pesquisadores parceiros dos Laboratórios de: Geomorfologia, Sedimentologia e Cartografia. 


\section{REFERÊNCIAS}

ABNT - Associação Brasileira de Normas Técnicas. NBR 6508 - Grãos de solo que passam na peneira 4,8mm - Determinação de massa específica - Métodos de ensaios, dezembro, outubro, 1984.

ABNT - Associação Brasileira de Normas Técnicas. NBR 7181 - Solo - Análise Granulométrica - Métodos de ensaios, dezembro, 1984.

ABNT - Associação Brasileira de Normas Técnicas. NBR 6457 - Amostras de solo Preparação para ensaios de compactação e ensaios de caracterização - Métodos de ensaios, agosto, 1986.

ACCORSI, O. J. A Exploração de areia para uso na construção civil: caracterização da atividade de dragagem e sustentabilidade na bacia hidrográfica do rio Acre. 2014. 187f. Tese de Doutorado em Engenharia Civil. Universidade Federal Fluminense (UFF), Niterói, 2014. Disponível em: http://icex.sites.uff.br/wpcontent/uploads/sites/461/2018/10/osmarformatada.pdf. Acesso em: 05 maio. 2020.

ANDERSSON, T. Estimating particle size distributions based on machine vision. (Thesis). Department of Computer Science and Electrical Engineering. Luleå University of Technology, 2010.

CHRISTOFOLETTI, A. Geomorfologia. 2 ed. São Paulo, Edgard. Voucher, 1980. 188p.

COX, E. A. Method of Assigning Numerical and Percentage Values to the Degree of Roundness of Sand Grains. Journal of Paleontology. n. 1, v. 3, 179 - 183, dez. 1927. Disponível em: http://www.jstor.org/stable/1298056. Acesso em: 02 maio 2020.

DIAS, J. A. A Análise sedimentar e o conhecimento dos sistemas marinhos. 2004. E-book disponível em: http://w3.ualg.pt/ jdias/JAD/eb_Sediment.html. Acesso em: 02 maio 2020.

GUERRA, A. T.; GUERRA, A. J. T. Novo Dicionário Geológico-Geomorfológico. $5^{\mathrm{a}}$ ed. Rio de Janeiro: Bertrand Brasil, 2006.

LANDINI, G. Operadores Morfológicos para ImageJ. (2006). Disponível em: http://www.mecourse.com/landinig/software/software.html. Acesso em: 03 maio 2020.

LEICA, M. FusionOptics: High Resolution and High Depth of Field Simultaneously. Disponível em: https://www.leica-microsystems.com. Acesso em: 02 jun. 2019.

MIRRA, A; MARCEL F; BRUNO, O. Análise Granulométrica (Dispersão Total): método da Pipeta. Instituto de Geociências - UFMG, 2014. 14p

PENTLAND, A. A method of measuring the angularity of sands. MAG. MN. A.L. Acta Eng. Dom. Transaction of the Royal Society of Canada, v. 21, 1927. 
POWERS, M. C. A new roundness scale for sedimentary particles. Journal of Sedimentary Petrology, v. 23, p.117-119. 1953.

RASBAND, W. S. ImageJ. National Institutes of Health, Bethesda, Maryland, USA, 2004. Disponível em: http://rsb.info.nih.gov/ij/. Acesso em: 03 maio 2020.

RIBEIRO, S.; BONETTI, C. Variabilidade morfométrica de sedimentos arenosos: revisão de métodos e uso do software ImageJ na diferenciação de ambientes deposicionais na Ilha de Santa Catarina e região continental, SC, Brasil. Revista GRAVEL. Porto Alegre, v.18, n.1, p. 38 46, dez. 2013. Disponível em: http://www.ufrgs.br/gravel/portugues/publica.htm. Acesso em: 02 maio 2020.

RILEY, N.A. Projection sphericity. Journal of Sedimentary Petrology, v. 11, n. 2, p. 94-97, 1941.

RODRIGUEZ, J. M; EDESKÄR, T; KNUTSSON, S. Particle Shape Quantities and Measurement Techniques-A Review. EJGE, v. 18, p. 169-198, 2013.

SUGUIO, K. Geologia sedimentar. São Paulo: Blucher, 2003. 383p.

SERRANO, R. O. P. [et. al.]. Variabilidade morfométrica dos sedimentos arenosos do rio Acre. In: CARVALHO, C. M. [et al.]. Ciência, inovação e tecnologia na Amazônia. 2. ed. Rio Branco: Stricto Sensu, p. 157-170, 2019. DOI: 10.35170/ss.ed.9786580261093.11. Acesso em: 07 maio 2020.

SERRANO, O. P.; CASTRO, A. L. P. de; RICO, E. A. M.; PINTO, M. A.; VIANA, E. M. de F.; MARTINEZ, C. B. Abrasive effects of sediments on impellers of pumps used for catching raw water. Agriambi, v. 22, n. 9, p. 591-596, 2018. DOI: DOI: http://dx.doi.org/10.1590/18071929/agriambi.v22n9p591-596.

TEIXEIRA, P. C. [et al.]. Manual de Métodos de Análise de Solo 3. ed. Brasília; Embrapa, 577p. 2017. Disponível em: https://www.infoteca.cnptia.embrapa.br/handle/doc/1085209. Acesso em: 02 maio 2020.

VIANA, J. H. M.; ClEMENTE, E. de P.; OLIVEIRA, A. P. de. Procedimento Operacional Padronizado para Quantificação e Mensuração de Areias Via Análise de Imagens. Rio de Janeiro, RJ: EMBRAPA, (Comunicado Técnico Online, 73), 9p., 2016. Disponível em: http://ainfo.cnptia.embrapa.br/digital/bitstream/item/160223/1/ComTec-73-QuantificacaoAreias.pdf. Acesso em: 02 maio 2020.

WADELL, H. Volume, Shape, and roundness of rock particles. Journal of Geology, v. 40, p. 443-451, 1932. 